\title{
Catastrophic health expenditure and its determinants in households with lung cancer patients in China: a retrospective cohort study
}

Cheng-yao Sun ${ }^{1}$, Ju-fang Shi ${ }^{2}$, Wen-qi Fu' ${ }^{1}$ Xin Zhang ${ }^{1}$, Guo-xiang Liu ${ }^{1 *}$, Wan-qing Chen ${ }^{2^{*}}$ and Jie He ${ }^{3^{*}}$

\begin{abstract}
Background: Numerous studies have examined catastrophic health expenditures (CHE) worldwide, mostly focusing on general or common chronic populations, rather than particularly vulnerable groups. This study assessed the medical expenditure and compensation of lung cancer, and explored the extent and influencing factors of CHE among households with lung cancer patients in China.
\end{abstract}

Methods: During 2018-2019, a hospital-based multicenter retrospective survey was conducted in seven provinces/ municipalities across China as a part of the Cancer Screening Program of Urban China. CHE was measured according to the proportion of out-of-pocket (OOP) health payments of households on non-food expenditures. Chi-square tests and logistic regression analysis was adjusted to determine the factors that significantly influenced the likelihood of a household with lung cancer patient to incur in CHE.

Results: In total, 470 households with lung cancer patients were included in the analysis. Health insurance was shown to protect some households from the impact of CHE. Nonetheless, CHE incidence (78.1\%) and intensity (14.02\% for average distance and $22.56 \%$ for relative distance) were still relatively high among households with lung cancer patients. The incidence was lower in households covered by the Urban Employee Basic Medical Insurance (UEMBI) insurance, with higher income level and shorter disease course.

Conclusion: More attention is needed for CHE incidence among vulnerable populations in China. Households with lung cancer patients were shown to be more likely to develop CHE. Therefore, policy makers should focus on improving the financial protection and reducing the economic burden of this disease.

Keywords: Catastrophic health expenditures, Insurance, Lung cancer, China

*Correspondence: Igx6301@163.com; chenwq@cicams.ac.cn; hejie@cicams. ac.cn

${ }^{1}$ Department of Health Economics, College of Health Management of Harbin Medical University, 157 Baojian Road, Harbin, People's Republic of China

${ }^{2}$ Office of Cancer Screening, National Cancer Center / National Clinical Research Center for Cancer / Cancer Hospital, Chinese Academy of Medical Sciences and Peking Union Medical College, Beijing 100021 China

${ }^{3}$ National Cancer Center/National Clinical Research Center for Cancer/ Cancer Hospital, Chinese Academy of Medical Sciences and Peking Union Medical College, Beijing, China

\section{Introduction}

Some of the fundamental roles of a healthcare system are protecting families from disease-related financial catastrophe and achieving health equality [1]. Hence, a high incidence of catastrophic health expenditures (CHE) means that a given health system is not achieving its goal of financial protection provision. In this context, a retrospective observational study conducted in 133 countries found that, within 5 years (i.e., from 2005 to 2010), CHE rose from 9.7 to $11.7 \%$ [2]. Another survey covering 89 countries worldwide showed that 150 million 
people face CHE annually, with low and middle income countries (LMICs) showing higher levels of CHE than high income countries [3]. Moreover, the World Health Survey showed that the incidence of CHE in developed countries, such as the United States of America and Germany, was $<1 \%$, while that in developing countries, such as Vietnam and Brazil, was $>10 \%$ [4]. Like many developing countries, China also faces a high disease burden and out-of-pocket (OOP) healthcare expenditure is relatively high; the incidence of CHE was about 8.94\% in 2016 [5].

Chronic diseases often come with high economic burdens. Many studies have shown that families with members who have chronic disease face higher financial risks than other families. In Bangalore, India, a study showed that $\mathrm{CHE}$ incidence in families with members who have chronic disease was $16 \%$, significantly exceeding the average level of the general population [6]. In rural China, families with members who have hypertension had 1.72.6 times higher occurrence of CHE than families without members with non-communicable diseases (NCDs) [7]. Another study reported that in China the incidence of $\mathrm{CHE}$ in families with members who have hypertension with other NCDs was $46.9 \%$ in 2013 [8], which significantly exceeded the average of $8.94 \%$.

Studies on CHE and its determinants have been conducted globally. However, most have focused on the whole population or common chronic populations, rather than on particularly vulnerable groups, such as cancer patients. In 2018, GLOBOCAN statistics found that, worldwide, there were 18.1 million new cancer cases and 9.6 million cancer deaths, with lung cancer being the most commonly diagnosed cancer and the leading cause of cancer death [9]. There were 3.804 million new cancer cases and 2.296 million deaths in 2014 across China, with lung cancer similarly being the most commonly diagnosed cancer (20\%) and the leading cause of cancer death (27.3\%) [10]. Owing to the high burden of morbidity and mortality related to lung cancer in China, we infer that it can give rise to $\mathrm{CHE}$ and impose a substantial financial burden in the Chinese population.

Health insurance has been widely accepted as an effective strategy to prevent CHE [11]. By 2015, more than 95\% of Chinese citizens participated in social health insurance [12]. The three types of social health insurance subsidized by the Chinese government are: Urban Employee Basic Medical Insurance (UEBMI), Urban Resident Basic Medical Insurance (URBMI), and the New Cooperative Medical Scheme (NCMS). The UEBMI was launched in 1998. Employers and employees jointly pay insurance premiums, and it covers urban employees and retirees in the formal sector, including those who previously enjoyed free medical care in public institutions and state-owned enterprises. The NCMS and URBMI were launched in 2003 and 2007, respectively. The former covers rural residents, and the latter covers urban residents who are not eligible for UEBMI, such as unemployed individuals and children. The reimbursement rates are the highest for the UEBMI, followed by the URBMI, and the NCMS [13].

Accordingly, the objective of this study was to assess the medical expenditure and compensation of lung cancer and explore the extent and influencing factors of $\mathrm{CHE}$ among households with lung cancer patients in China. Furthermore, this study evaluated the financial protection capacity of health insurance for families with lung cancer patients. Our findings may contribute to improving and adjusting related medical insurance policy, helping to further relieve the economic burden of this critical disease.

\section{Methods and materials \\ Data source}

The Cancer Screening Program in Urban China (CanSPUC), which was supported by the National Health and Family Planning Commission (NHFPC) in China, has been a crucial cancer prevention initiative that began in August 2012 [14]. The primary objective of the CanSPUC was to explore an appropriate implementation approach for screening of population at high risk and early diagnosis of major cancers in urban populations in China, promoting the use of mature screening and early diagnosis technology for common cancers to reduce mortalities. The program covered 13 provinces/municipalities in China [including eastern (Beijing, Hebei, Liaoning, Jiangsu, Zhejiang, Shandong, and Guangdong), central (Heilongjiang, Henan, and Hunan), and western regions (Chongqing, Gansu, and Xinjiang) [15] by 2014.

A multicenter cross-sectional survey was conducted from January 2018 to June 2019 as part of CanSPUC. Geographic regions/provinces were grouped into eastern, central and western in line with the classification of economic development zones by the Chinese National Bureau of Statistics. Nine tertiary hospitals, were selected from these zones considering cancer patient volumes and completeness of medical records, including Guangdong Cancer Hospital (eastern); Anhui Cancer Hospital, Heilongjiang Cancer Hospital, Shanxi Cancer Hospital (central); Guangxi Cancer Hospital, Yunnan Cancer Hospital, the Regional Cancer Hospital and two city hospitals in Inner Mongolia (western).

Cancer patients initially diagnosed between 01 January 2015 and 31 December 2016 were eligible for this study (including lung, female breast, colorectal, esophageal, gastric, and liver cancers). Eligible study participants were identified from the hospital records and then approached for a survey. Upon consent to participate in 
the study, an informed consent form was completed by the patient. The questionnaire was administered through face-to-face interviews. The survey was coordinated by the National Cancer Center. The interviewers were trained prior to deployment and required to check completeness of the questionnaire before concluding each interview. The questionnaire for this survey collected data regarding demographic characteristics, household income and expenditure, medical expenses for cancer treatment, and insurance compensation. A total of 2565 patients being investigated.

Regarding household income and expenditure, respondents were asked to describe them for both 2015 and 2016. Regarding medical expenses for cancer treatment, when the course of the patient's disease was 1 year, the respondents were asked to describe this variable over a one-year period (i.e., 2 months before and 10 months after the diagnosis); when the course of the patient's disease was 2 years, the respondents were asked to describe this variable over a two-year period (i.e., 2 months before and 22 months after the diagnosis); these costs included payments for hospital diagnosis, treatment, and medicines (prescription and non-prescription drugs) purchased from pharmacy retail stores.

Among these, lung cancer patients were selected as subjects of this study. Inclusion criteria were as follows: (1) having been diagnosed for the first time with primary lung cancer, (2) having been initially diagnosed between 1 January 2015 and 31 December 2016, and (3) having subsequently received cancer treatment. The exclusion criterion was having cancers in multiple organs. Regarding household income and expenditure, owing to difficulties in articulating a clear cut-off point for the income and expenditure data, we calculated average income and expenditure across the 2 years to match the lung cancer treatment cost data. Regarding medical expenses, when the course of disease was 2 years, we calculated the average medical expenses across 2 years. Chinese Renminbi (RMB) was converted into US dollars based on the average 2015 exchange rate (Chinese RMB 6.2284 yuan $=\$$ US1.00).

Data were double-entered into EpiData 3.1 to ensure accuracy and analyzed using Stata 15 .

\section{Measuring CHE incidence}

Two thresholds have been widely used to define CHE: OOP healthcare expenditure greater than or equal to $10 \%$ of total household expenditure [16, 17]; or a non-food household expenditure greater than or equal to $40 \%$ [18, 19]. We measured CHE using the indicators reported by Adam et al. [20]. In this study, OOP health expenditure only covered direct medical expenses, excluding direct non-medical (e.g., transportation and nutrition) and indirect costs. To calculate CHE, we used non-food household expenditure as the denominator, thereby partly avoiding measurement bias found in other methods that often cause the neglect of low income families. In the equation below, the indicator $E i$ defined whether CHE occurred:

$$
E_{i}=\left\{\begin{array}{l}
0, \frac{o o p}{e_{i}-f_{i}}<z \\
1, \frac{o o p}{e_{i}-f_{i}}>z
\end{array}\right.
$$

where $e_{i}$ is the total consumption expenditure of household $i$, and $f_{i}$ is the food expenditure of household $i$. Before insurance compensation, the OOP is the total medical cost (including insurance compensation). The $e_{i}$ is the total consumption expenditure of household $i$ (including insurance compensation). After insurance compensation, the OOP is the patient's medical costs after reimbursement. The $e_{i}$ is the total household expenditure of household $i$ after insurance compensation is deleted. $\mathrm{z}$ is the threshold of CHE, which we set to 0.4. $\mathrm{CHE}$ incidence and intensity were estimated as follows:

$$
\begin{aligned}
& \mathrm{H}=1 / \mathrm{N} \sum_{i=1}^{N} E i \\
& \mathrm{O}=1 / \mathrm{N} \sum_{i=1}^{N}\left(\left(\frac{o o p}{e i-f i}\right)-z\right) * E i \\
& \mathrm{MPO}=1 / \mathrm{Na} \sum_{i-1}^{N a}\left(\left(\frac{o o p}{e i-f i}\right)-z\right) * E i
\end{aligned}
$$

where $\mathrm{N}$ is the sample size; $\mathrm{Na}$ is the sample size of households incurring in CHE; $\mathrm{H}$ is CHE incidence; $\mathrm{O}$ is the average distance; and MPO is the mean relative distance. CHE intensity was calculated using average distance and mean relative distance: average distance measures the degree by which an average OOP health expenditure exceeds the given CHE threshold of all lung cancer families; and mean relative distance represents CHE intensity in families suffering from CHE.

\section{Statistical analysis}

Medical expenditure, insurance compensation, household income and expenditure, and OOP were presented using means and standard deviations. The analysis of the collected data was done while stratifying the variables by two different insurance groups: the UEBMI group and the Urban and Rural Resident Basic Medical Insurance (U\&RRBMI) group (U\&RRBMI includes URBMI and NCMS).

We used the chi-squared test to compare the CHE incidence in lung cancer families before and after insurance compensation. In addition, the chi-squared test was 
applied to examine the associations between $\mathrm{CHE}$ and other variables including gender, age, education level, household size, insurance, and income level. Additionally, multivariate logistic regression analyses were performed and $P<.05$ was considered statistically significant.

Econometricians usually dismissed sample size problems based on the strength of the asymptotic quality of the standard maximum likelihood (ML) model [21]. However, others made some suggestions regarding sample size; Hart and Clark found that reasoning problems began to occur when the sample size was less than 30 . In another study with a sample of 200 participants, Hart concluded that this sample produced a consistent estimate of the probit model [22]. Eliaison additionally recommended that the sample size should be more than 60 [23]. Therefore, to meet the model requirements, we deemed a sample size of 470 lung cancer families to be adequate.

\section{Results}

\section{Sample characteristics}

A total of 470 households with a lung cancer patient were recruited. Table 1 shows the general characteristics of lung cancer patients and their families. In total, $63 \%$ of the lung cancer patients were male and the sample showed a rather high average age at 63.6. Regarding education, most graduated from junior high school and below (61.3\%), with $38.7 \%$ having graduated from senior high school. Moreover, $86.6 \%$ of the respondents were married. The course of disease revealed that $83.2 \%$ had cancer for 1-2 years. Regarding household size, $59.4 \%$ of the lung cancer families had less than 4 people and $35.7 \%$ had 4-6 people, together totalizing 95.1\%. Regarding type of health insurance, most respondents were covered by health insurance policies, with UEBMI accounting for $54.7 \%$ and U\&RRBMI for $44 \%$.

The mean annual household expenditure of the UEBMI and U\&RRBMI groups were $\$ 11,366(\mathrm{SD}=8255)$ and $\$ 9835(\mathrm{SD}=8993)$, respectively (Table 2). The annual medical household expenditure of the UEBMI and U\&RRBMI groups were $\$ 13,527 \quad(\mathrm{SD}=10,265)$ and $\$ 12,689$ ( $\mathrm{SD}=8962)$, respectively. The average insurance compensation provided to the UEBMI and U\&RRBMI groups were $\$ 6633(\mathrm{SD}=6038)$ and $\$ 4671(\mathrm{SD}=3888)$, respectively. The mean OOP expenditure for lung cancer care of the UEBMI and U\&RRBMI groups were $\$ 6674$ $(\mathrm{SD}=6519)$ and $\$ 7892(\mathrm{SD}=6967)$, respectively.

\section{CHE and the financial protection capacity of health insurance}

Figure 1 and Table 3 show the CHE incidence and intensity of lung cancer families before and after insurance compensation. The CHE incidence of lung
Table 1 Participant characteristics

\begin{tabular}{|c|c|c|}
\hline Sociodemographic Characteristics & Number & Percent (\%) \\
\hline \multicolumn{3}{|l|}{ Gender } \\
\hline Male & 296 & 63.00 \\
\hline Female & 174 & 37.00 \\
\hline \multicolumn{3}{|l|}{ Age (years) } \\
\hline$\leq 60$ & 186 & 39.60 \\
\hline$>60$ & 284 & 60.40 \\
\hline \multicolumn{3}{|l|}{ Education level } \\
\hline Junior high school and below & 288 & 61.30 \\
\hline Senior high school and above & 182 & 38.70 \\
\hline \multicolumn{3}{|l|}{ Marital status } \\
\hline Married & 407 & 86.60 \\
\hline Rest $^{1}$ & 63 & 13.40 \\
\hline \multicolumn{3}{|l|}{ Course of disease } \\
\hline$<1$ year & 79 & 16.80 \\
\hline 1-2year & 391 & 83.20 \\
\hline \multicolumn{3}{|l|}{ Household size } \\
\hline$\leq 3$ & 279 & 59.40 \\
\hline $4-6$ & 168 & 35.70 \\
\hline$\geq 7$ & 23 & 4.90 \\
\hline \multicolumn{3}{|l|}{ Insurance } \\
\hline UEBMI & 257 & 54.70 \\
\hline U\&RRBMI & 207 & 44.00 \\
\hline Rest $^{2}$ & 6 & 1.30 \\
\hline \multicolumn{3}{|l|}{ Income level(80000RMB) } \\
\hline$\leq 12844$ USD & 346 & 73.60 \\
\hline$>12844$ USD & 124 & 26.40 \\
\hline
\end{tabular}

Rest 1 represents unmarried, divorced and widowed. Rest 2 stands for public medical, commercial insurance and uninsured. Abbreviations: The UEBMI represents Urban Employee Basic Medical Insurance. The U\&RRBMI represents Urban and Rural Resident Basic Medical Insurance

cancer families before insurance compensation was 93 and $78.10 \%(p=0.000)$ after insurance compensation, indicating that insurance compensation reduced $\mathrm{CHE}$ incidence by $14.9 \%$. Meanwhile, the CHE incidence of lung cancer families covered by UEBMI were 91.4 and $73.2 \%(p=0.000)$ before and after insurance compensation, respectively; namely, UEBMI insurance compensation reduced $\mathrm{CHE}$ incidence by $18.2 \%$. The CHE incidence of lung cancer families covered by U\&RRBMI were 94.69 and $84.06 \%(p=0.000)$ before and after insurance compensation, respectively; hence, U\&RRBMI insurance compensation reduced the incidence of $\mathrm{CHE}$ by $10.63 \%$.

The relative distance was $22.56 \%$ after insurance compensation, indicating that families which suffered from CHE had an average of $62.56 \%$ of their household expenditure net of food spending characterized by OOP expenditure. Furthermore, the relative distance was $10.86 \%$ in lung cancer families covered by UEBMI (i.e., 
Table 2 Medical expenditure, compensation and OOP payment by insurance type

\begin{tabular}{|c|c|c|c|c|c|c|}
\hline \multirow[b]{2}{*}{ Indicators } & \multicolumn{2}{|l|}{ UEBMI } & \multicolumn{2}{|c|}{ U\&RRBMI } & \multicolumn{2}{|l|}{ Total } \\
\hline & Mean & SD & Mean & SD & Mean & SD \\
\hline Medical expenditure & 13,527 & 10,265 & 12,689 & 8962 & 13,173 & 9677 \\
\hline Insurance reimbursement & 6633 & 6038 & 4671 & 3888 & 5755 & 5258 \\
\hline OOP payment & 6674 & 6519 & 7892 & 6967 & 7242 & 6768 \\
\hline Household income & 14,362 & 11,607 & 7907 & 8248 & 11,473 & 10,697 \\
\hline Household expenditure & 11,366 & 8255 & 9835 & 8993 & 10,733 & 8618 \\
\hline Food expenditure & 3027 & 2277 & 1872 & 1855 & 2509 & 2167 \\
\hline
\end{tabular}

Abbreviations: The UEBMI represents Urban Employee Basic Medical Insurance. The U\&RRBMI represents Urban and Rural Resident Basic Medical Insurance

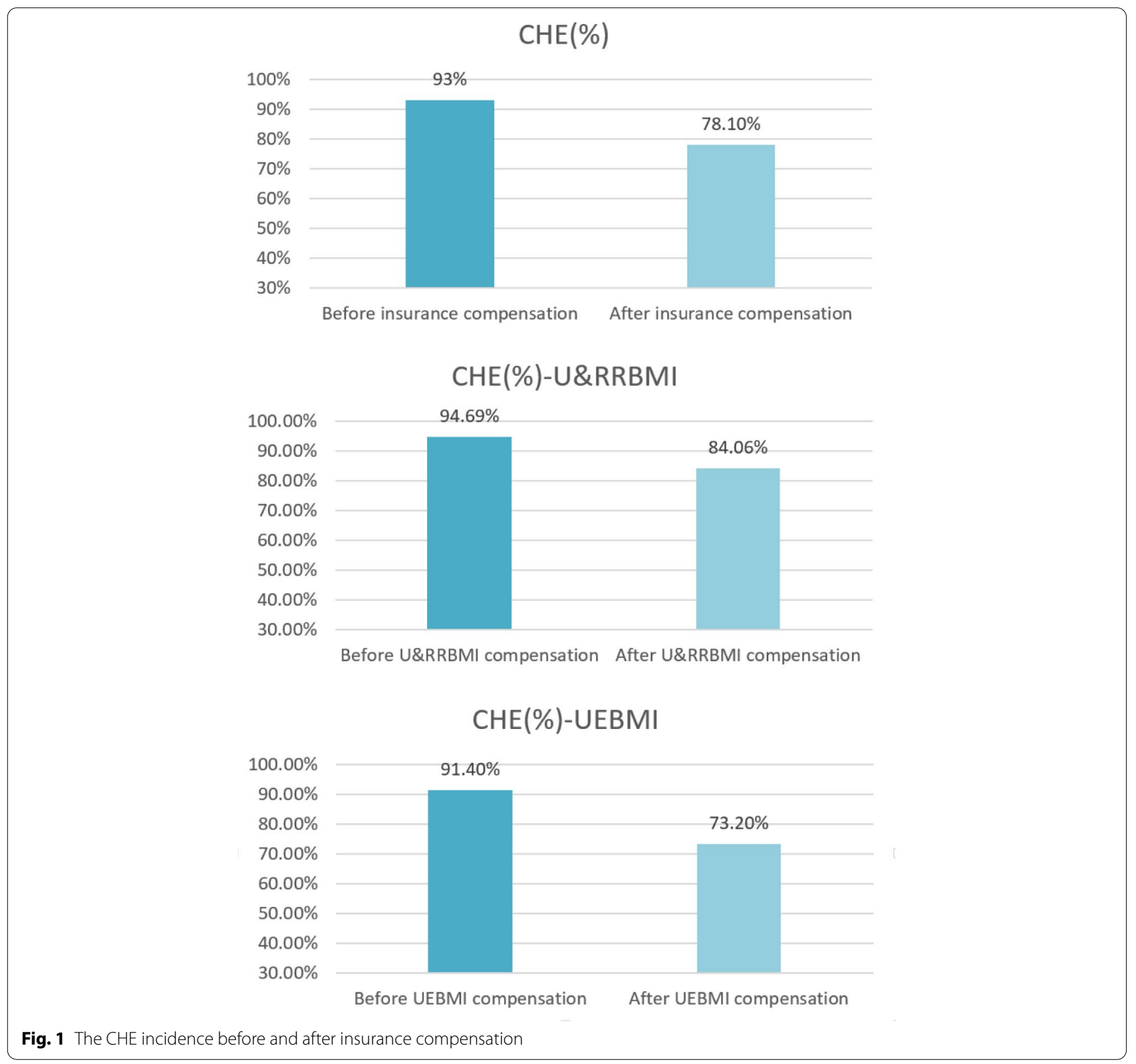


Table 3 the CHE incidence and intensity before and after insurance compensation

\begin{tabular}{|c|c|c|c|c|}
\hline & Average distance & Reduced & Relative distance & Reduced \\
\hline \multicolumn{5}{|l|}{ Total } \\
\hline Before compensation & $23.80 \%$ & & $27.70 \%$ & \\
\hline After compensation & $14.02 \%$ & $9.78 \%$ & $22.56 \%$ & $5.14 \%$ \\
\hline \multicolumn{5}{|l|}{ UEBMI } \\
\hline Before compensation & $21.68 \%$ & & $26.53 \%$ & \\
\hline After compensation & $10.96 \%$ & $10.72 \%$ & $10.86 \%$ & $15.67 \%$ \\
\hline \multicolumn{5}{|l|}{ U\&RRBMI } \\
\hline Before compensation & $26.28 \%$ & & $28.94 \%$ & \\
\hline After compensation & $17.68 \%$ & $8.6 \%$ & $24.08 \%$ & $4.86 \%$ \\
\hline
\end{tabular}

Abbreviations: The UEBMI represents Urban Employee Basic Medical Insurance. The U\&RRBMI represents Urban and Rural Resident Basic Medical Insurance

this insurance reduced the relative distance by $15.67 \%)$ and $24.08 \%$ in those covered by U\&RRBMI (i.e., this insurance reduced the relative distance by $4.86 \%$ ). The average distance of lung cancer families was $14.02 \%$ after insurance compensation, with $10.96 \%$ for lung cancer families covered by UEBMI and $17.68 \%$ for those covered by U\&RRBMI.

Patients with lung cancer who had longer course of disease, U\&RRBMI insurance, lower income level tended to have higher incidence of CHE than the others (Table 4). The logistic regression model further confirmed that course of disease, insurance, age, and income level were significant predictors of CHE. (Table 5).

\section{Discussion}

To the best of our knowledge, this was the first study to analyze the impact of insurance on and the extent and determinant factors of $\mathrm{CHE}$, as well as the medical expenditure and insurance compensation in households with members who have lung cancer patients in China.

Our findings showed that the mean medical expenditure of lung cancer patients was $\$ 13,173$, which is much higher than the gross domestic product (GDP) per capita in China (\$7904 in 2015) [24]. The average OOP payment after insurance compensation for lung cancer patients was $\$ 7242$, being nearer to the aforementioned GDP per capita. The medical expenditure of those covered by UEBMI was higher than that of those covered by U\&RRBMI, albeit the OOP payment of the first was lower than that of the second; this owes to the higher percentage of insurance reimbursement of the UEBMI insurance. The household expenditure was higher in those covered by UEBMI than by U\&RRBMI. Therefore, OOP health expenditure brought about a huge financial burden to the households with members who have lung cancer, especially those covered by U\&RRBMI insurance. This finding is consistent with previous studies $[25,26]$.
Table 4 Incidence of CHE after insurance compensation

\begin{tabular}{|c|c|c|c|}
\hline Sociodemographic Characteristics & $\mathrm{CHE}(\mathrm{n})$ & Percent (\%) & $P$ \\
\hline \multicolumn{4}{|l|}{ Gender } \\
\hline Male & 236 & $79.73 \%$ & \multirow[t]{2}{*}{0.261} \\
\hline Female & 131 & $75.29 \%$ & \\
\hline \multicolumn{4}{|l|}{ Age (years) } \\
\hline$\leq 60$ & 142 & $76.34 \%$ & \multirow[t]{2}{*}{0.460} \\
\hline$>60$ & 225 & $79.23 \%$ & \\
\hline \multicolumn{4}{|l|}{ Education level } \\
\hline Junior high school and below & 229 & $79.51 \%$ & \multirow[t]{2}{*}{0.346} \\
\hline Senior high school and above & 138 & $75.82 \%$ & \\
\hline \multicolumn{4}{|l|}{ Marital status } \\
\hline Married & 319 & $78.38 \%$ & \multirow[t]{2}{*}{0.696} \\
\hline Rest1 & 48 & $76.19 \%$ & \\
\hline \multicolumn{4}{|l|}{ Course of disease } \\
\hline$<1$ year & 44 & $55.70 \%$ & \multirow[t]{2}{*}{0.000} \\
\hline $1-2$ year & 323 & $82.61 \%$ & \\
\hline \multicolumn{4}{|l|}{ Household size } \\
\hline$\leq 3$ & 218 & $78.14 \%$ & \multirow[t]{3}{*}{0.580} \\
\hline $4-6$ & 133 & $79.17 \%$ & \\
\hline$\geq 7$ & 16 & $69.57 \%$ & \\
\hline \multicolumn{4}{|l|}{ Insurance } \\
\hline UEBMI & 188 & $73.15 \%$ & \multirow[t]{3}{*}{0.018} \\
\hline U\&RRBMI & 174 & $84.06 \%$ & \\
\hline Rest2 & 5 & $83.33 \%$ & \\
\hline \multicolumn{4}{|l|}{ Income level(80,000 RMB) } \\
\hline$\leq 12844$ USD & 289 & $83.53 \%$ & \multirow[t]{2}{*}{0.000} \\
\hline$>12844$ USD & 78 & $62.90 \%$ & \\
\hline
\end{tabular}

P based on chi-square test. Abbreviations: The UEBMI represents Urban Employee Basic Medical Insurance. The U\&RRBMI represents Urban and Rural Resident Basic Medical Insurance

Additionally, we found that $78.10 \%$ of the households with lung cancer patients demonstrated health expenditure that went above $40 \%$ of their non-food expenditures. In another Chinese study, the overall CHE incidence was 13.0\% (the 
Table 5 Multivariate logistic regression model of determinants of CHE

\begin{tabular}{|c|c|c|c|c|c|}
\hline Determinants & OR & SE & $\mathbf{p}$ & $95 \% \mathrm{Cl}$ & \\
\hline \multicolumn{6}{|l|}{ Gender (ref male) } \\
\hline Female & 0.874 & 0.225 & 0.599 & 0.528 & 1.446 \\
\hline \multicolumn{6}{|c|}{ Age (years, ref $\leq 60$ ) } \\
\hline$>60$ & 2.162 & 0.591 & 0.005 & 1.266 & 3.693 \\
\hline \multicolumn{6}{|c|}{ Education level (ref Junior high school and below) } \\
\hline $\begin{array}{c}\text { Senior high } \\
\text { school and above }\end{array}$ & 1.278 & 0.365 & 0.390 & 0.730 & 2.239 \\
\hline \multicolumn{6}{|c|}{ Marital status (ref Married) } \\
\hline Rest $^{1}$ & 0.827 & 0.302 & 0.604 & 0.404 & 1.693 \\
\hline \multicolumn{6}{|c|}{ Course of disease (ref $<1$ year) } \\
\hline $1-2$ year & 5.692 & 1.725 & 0.000 & 3.143 & 10.308 \\
\hline \multicolumn{6}{|c|}{ Household size (ref $\leq 3$ ) } \\
\hline $4-6$ & 1.024 & 0.279 & 0.930 & 0.601 & 1.746 \\
\hline$\geq 7$ & 0.444 & 0.238 & 0.129 & 0.155 & 1.268 \\
\hline \multicolumn{6}{|c|}{ Insurance (ref UEMBI) } \\
\hline U\&RRBMI & 2.262 & 0.664 & 0.005 & 1.272 & 4.023 \\
\hline Rest2 & 1.656 & 1.948 & 0.668 & 0.165 & 16.612 \\
\hline \multicolumn{6}{|c|}{ Income level (ref $\leq 12,844$ USD) } \\
\hline$>12,844$ USD & 0.292 & 0.081 & 0.000 & 0.170 & 0.503 \\
\hline Constant & 0.637 & 0.261 & 0.272 & 0.285 & 1.423 \\
\hline
\end{tabular}

Rest 1 represents unmarried, divorced and widowed. Rest 2 stands for public medical, commercial insurance and uninsured. Abbreviations: The UEBMI represents Urban Employee Basic Medical Insurance. The U\&RRBMI represents Urban and Rural Resident Basic Medical Insurance

threshold is set to 40\%) [27]. In another study, $21.5 \%$ of the families with members who had only hypertension and $46.9 \%$ of the families with members who had hypertension plus other NCDs incurred in CHE [8]. Namely, in China, lung cancer households' risk tolerance for health expenditure may be much lower than the average for the general population or for households with other chronic diseases.

Moreover, our findings showed that the studied households had a lower CHE incidence after insurance compensation than before it; namely, the studied health insurance systems (i.e., UEMBI and U\&RRBMI) protected some households from the impact of CHE. We also found that the impact of UEMBI on CHE was greater than that of U\&RRBMI. However, even if provided relevant data on the difference in compensation levels between these two insurances, we still see a need for further study on the overall incidence of CHE in lung cancer families; given that our findings showed that lung cancer families may be one of the most vulnerable groups to $\mathrm{CHE}$, future research should pay more attention to such households and study the incidence of this issue.

In this study, increased disease course, low-level insurance, age over 60 years, and low-level income significantly impacted CHE; these findings find consonance in the literature. A study examining the determinants factors of CHE among cancer families across 10 countries in Southeast Asia found that income level, education, and type of health institution and health insurance are influencing factors of CHE [28], hence corroborating our findings. Another study demonstrated that Chinese older adults' risk tolerance for healthcare payments was actually lower than the average in China [29]. Therefore, to help mitigate CHE risk in households with lung cancer patients, we suggest for the central government to adjust the related policies by age, as there seems to be age differences on the topic.

In 2009, China's health reforms focused on reducing OOP spending. Catastrophic medical insurance was introduced in 2012 to cover more than 4 million patients with catastrophic illnesses [30]. However, CHE for lung cancer patients remains high even after the implementation of catastrophic insurance. According to our findings, the CHE rates for households with lung cancer patients leads to high medical costs. The rapid growth of the national health expenditure per capita is an important cause of CHE. The National Bureau of Statistics showed that the national health expenditure per capita increased from 1314 Yuan in 2009 to 4236 Yuan in 2018 [31]. Therefore, policy-makers should endeavor to develop policies that help control the cost of medical procedures. Additionally, China's catastrophic medical insurance is operated by commercial insurance companies. The catastrophic medical insurance is not perfect and has not played the role it should have played.

Chinese hospitals are organized according to a threetier system that recognizes the ability to provide medical care, education, and conduct medical research [15]. Based on this system, hospitals are designated as primary, secondary, or tertiary institutions, and the medical expenses tend to be higher in high-level hospitals. However, owing to the limited capacity of medical care in primary hospitals, many patients choose to go to high-level hospitals. Therefore, to reduce patients' financial burden related to health expenditure, the resource allocation of the three-tier system should be optimized and aim to avoid congregating patients in tertiary hospitals.

Some limitations of our study must be acknowledged. First, we evaluated CHE incidence and intensity only in households with lung cancer patients that actually presented themselves for treatment, not considering households that did not receive treatment. Many patients decide not to receive treatment owing to, for instance, insufficient family funds or thinking that the treatment will not save their lives. Second, OOP health expenditure in this study only covered direct medical expenditures, excluding direct non-medical (e.g., transportation and nutrition) and indirect costs. Therefore, $\mathrm{CHE}$ incidence might have been underestimated 
to some extent. Third, the sample size of lung cancer households was not very large. Therefore, it is recommended for future studies to recruit a larger sample. Fourth, the severity of lung cancer is also the impact factor of financial burden [32]. In the future, we will take into account variables related to disease severity such as cancer staging and treatment methods to explore the influencing factors of $\mathrm{CHE}$ in a more comprehensive manner. Finally, the findings of this study were based on patients in tertiary hospitals in an urban setting; hence, the conclusion should not be overstated.

\section{Conclusions}

The findings revealed that CHE incidence and intensity were relatively high among households with lung cancer patients. Furthermore, more attention is warranted to households covered by U\&RRBMI because they seemed to be, based on our findings, at the highest risk of incurring CHE. Moreover, some social factors significantly affected CHE, meaning that policies aimed at reducing CHE must consider some of the described social factors of households and patients.

\begin{abstract}
Abbreviations
CHE: Catastrophic health expenditures; OOP: Out-of-pocket; UEMBI: Urban Employee Basic Medical Insurance; NCDs: Non-communicable diseases; URBMI: Urban Resident Basic Medical Insurance; NCMS: New Cooperative Medical Scheme; CanSPUC: The Cancer Screening Program in Urban China; NHFPC: National Health and Family Planning Commission; RMB: Renminbi; MPO: Mean relative distance; O: Average distance; U\&RRBMI: Urban and Rural Resident Basic Medical Insurance; GDP: Gross domestic product.
\end{abstract}

\section{Acknowledgements}

The authors would like to thank each patient with lung cancer and wish them good health.

\section{Authors' contributions}

Acquisition of data and conceived the research idea: $\mathrm{H}$ and W-qC. Conception and design; analysis and interpretation of data: J-fS, W-qF and XZ. Writing and drafting of the manuscript; Analysis and interpretation of data; statistical analysis: C-yS. Critical revision of the manuscript for important intellectual content, and conceived the research idea: G-xL. All authors have read and approved the final manuscript.

\section{Funding}

This work was supported by National Key R\&D Program of China (2017YFC13 08700/2017YFC1308705); the National Natural Science Foundation of China (71673071); the National Key Public Health Program of China (Cancer Screening Program in Urban China).

The funders played a role in publication of study findings.

\section{Availability of data and materials}

The data that support the findings of this study are available from Chinese Academy of Medical Sciences. The datasets used in this study were available from the corresponding author upon reasonable request.

\section{Declarations}

Ethics approval and consent to participate

The study protocol followed the tenets of the declaration of Helsinki. This study was reviewed and approved by the Ethics Committee of the Cancer
Institute and Hospital, Chinese Academy of Medical Sciences (15-070/997). Written informed consent was obtained from all participants.

\section{Consent for publication}

Not applicable.

\section{Competing interests}

The authors declare that there are no competing interests.

Received: 2 June 2021 Accepted: 17 November 2021

Published online: 10 December 2021

\section{References}

1. Marmot $\mathrm{M}$, et al. Closing the gap in a generation: health equity through action on the social determinants of health. Lancet. 2008. https://doi.org/ 10.1016/S0140-6736(08)61690-6.

2. Wagstaff A, Flores G, Hsu J, Smitz MF, Chepynoga K, Buisman LR, van Wilgenburg K, Eozenou P. Progress on catastrophic health spending in 133 countries: a retrospective observational study. Lancet Glob Health. 2018;6(2):e169-e179. https://doi.org/10.1016/S2214-109X(17)30429-1.

3. Xu K, et al. Protecting households from catastrophic health spending. Health Aff. 2007:26(4):972

4. Xu K, et al. Assessing the reliability of household expenditure data: results of the world health survey. Health Policy. 2009;91(3):297-305.

5. Ma X, Wang Z, Liu X. Progress on Catastrophic Health Expenditure in China: Evidence from China Family Panel Studies (CFPS) 2010 to 2016. Int J Environ Res Public Health. 2019;16(23). https://doi.org/10.3390/ijerph16234775.

6. Bhojani U, Thriveni B, Devadasan R, Munegowda C, Devadasan N, Kolsteren P, Criel B. Out-of-pocket healthcare payments on chronic conditions impoverish urban poor in Bangalore, India. BMC Public Health. 2012;12:990. https://doi.org/10.1186/1471-2458-12-990.

7. Liu $X$, et al. Financial protection of rural health insurance for patients with hypertension and diabetes: repeated cross-sectional surveys in rural China. BMC Health Serv Res. 2016;16(1):481.

8. Si Y, Zhou Z, Su M, Wang X, Lan X, Wang D, Gong S, Xiao X, Shen C, Ren Y, Zhao D, Hong Z, Bian Y, Chen X. Decomposing inequality in catastrophic health expenditure for self-reported hypertension household in Urban Shaanxi, China from 2008 to 2013: two waves' cross-sectional study. BMJ Open. 2019;9(5):e023033. https://doi.org/10.1136/bmjopen-2018-023033.

9. Bray F, Ferlay J, Soerjomataram I, Siegel RL, Torre LA, Jemal A. Global cancer statistics 2018: GLOBOCAN estimates of incidence and mortality worldwide for 36 cancers in 185 countries. CA Cancer J Clin. 2018;68(6):394-424. https://doi.org/10.3322/caac.21492.

10. Chen W, et al. Cancer incidence and mortality in China, 2014. Chin J Cancer Res. 2018;030(001):1-12.

11. Rossell $N$, et al. Choosing a miracle: Impoverishment, mistrust, and discordant views in abandonment of treatment of children with cancer in El Salvador. Psychooncology. 2017;26(9):1324-9. https://doi.org/10.1002/pon.4302.

12. Sun J, Lyu S. The effect of medical insurance on catastrophic health expenditure: evidence from China. Cost Eff Resour Alloc. 2020;18:10. https://doi.org/10.1186/s12962-020-00206-y.

13. Fu W, et al. Effects of cancer treatment on household impoverishment: a multicentre cross-sectional study in China. BMJ Open. 2021;11(6):e044322. https://doi.org/10.1136/bmjopen-2020-044322.

14. Dai M, Jufang S, Li N. The design and expected goal for cancer screening program in urban China. Chin J Prev Med. 2013;47(2):179-82 (in Chinese).

15. Ministry of Health of the People's Republic of China. China Health statistics yearbook 2012. Beijing: China Union Medical University Press; 2012. Accessed 20 Mar 2017 (in Chinese)

16. Bredenkamp C, Mendola M. Catastrophic and impoverishing effects of health expenditure: new evidence from the Western Balkans. Health Policy Plan. 2011;26(4):349.

17. Galárraga $\mathrm{O}$, et al. Health insurance for the poor: impact on catastrophic and out-of-pocket health expenditures in Mexico. Eur J Health Econ. 2010;11(5):437-47.

18. Onwujekwe $\mathrm{O}$, Hanson $\mathrm{K}$, Uzochukwu B. Examining inequities in incidence of catastrophic health expenditures on different healthcare services and health facilities in Nigeria. PLoS One. 2012;7(7):e40811. https:// doi.org/10.1371/journal.pone.0040811. 
19. Van Minh H, Kim Phuong NT, Saksena P, James CD, Xu K. Financial burden of household out-of pocket health expenditure in Viet Nam: findings from the National Living Standard Survey 2002-2010. Soc Sci Med. 2013;96:258-63. https://doi.org/10.1016/j.socscimed.2012.11.028.

20. Wagstaff $A$, van Doorslaer E. Catastrophe and impoverishment in paying for health care: with applications to Vietnam 1993-1998. Health Econ. 2003:12(11):921-34. https://doi.org/10.1002/hec.776.

21. Webel K, Greene WH. Econometric analysis. Statal Papers. 2011;52(4):983-4.

22. Hart RA, Clark DH. Does size matter? Exploring the small sample properties of maximum likelihood estimation. In: Annual Meeting of the Midwest Political Science Association; 1999.

23. Eliason SR. Maximum likelihood estimation : logic and practice. J Am Stat Assoc. 1993;89(427):1150

24. National Bureau of Statistics. Available from: https://data.stats.gov.cn/ easyquery.htm?cn $=C 01 \& z b=A 0201 \& s j=2020$

25. Migliorino MR, et al. Economic burden of patients affected by nonsmall cell lung cancer (NSCLC): the LIFE study. J Cancer Res Clin Oncol. 2017;143(5):783-91. https://doi.org/10.1007/s00432-016-2326-x.

26. Zhang $X$, et al. Economic Burden for Lung Cancer Survivors in Urban China. Int J Environ Res Public Health. 2017;14(3). https://doi.org/10.3390/ ijerph14030308.

27. $\mathrm{Wu} \mathrm{QH}$, et al. Effect of health insurance on reduction of catastrophic health expenditure in China. Chin J Health Policy. 2012;9:62-6.

28. TAS., G. Catastrophic health expenditure and 12-month mortality associated with cancer in Southeast Asia: results from a longitudinal study in eight countries. BMC Med. 2015;13(1):1-11.

29. Wang $Z, L i X$, Chen M. Catastrophic health expenditures and its inequality in elderly households with chronic disease patients in China. Int J Equity Health. 2015;14(1):8.

30. Li H, Jiang L. Catastrophic medical insurance in China. Lancet. 2017;390(10104):1724-5. https://doi.org/10.1016/s0140-6736(17)32603-x.

31. National Bureau of Statistics. Available from: http://www.stats.gov.cn/.

32. Zhang $X$, et al. Medical expenditure for lung cancer in China: a multicenter, hospital-based retrospective survey. Cost Eff Resour Alloc. 2021;19(1):53. https://doi.org/10.1186/s12962-021-00306-3.

\section{Publisher's Note}

Springer Nature remains neutral with regard to jurisdictional claims in published maps and institutional affiliations.

Ready to submit your research? Choose BMC and benefit from:

- fast, convenient online submission

- thorough peer review by experienced researchers in your field

- rapid publication on acceptance

- support for research data, including large and complex data types

- gold Open Access which fosters wider collaboration and increased citations

- maximum visibility for your research: over $100 \mathrm{M}$ website views per year

At BMC, research is always in progress.

Learn more biomedcentral.com/submissions 